\title{
Simultaneous correction of high order geometrical driving terms with octupoles in synchrotron light sources
}

\author{
Fabien Plassard๑," Guimei Wang®, Timur Shaftan, Victor Smaluk®, \\ Yongjun Li, and Yoshiteru Hidaka \\ Brookhaven National Laboratory, Upton, New York 11973, USA
}

(Received 8 September 2021; accepted 1 November 2021; published 24 November 2021)

\begin{abstract}
As the next generation of light sources is pushing toward high-brightness storage rings with ultralow emittance, the control of the beam nonlinear dynamics becomes increasingly challenging. Nonlinear perturbations, arising from sextupole cross talks, set the limit to the achievable dynamic performance of the machine. Octupoles can be an efficient mean to tackle the remaining resonant driving terms generated to the second order by the lattice sextupoles. However, they have been used sparingly in light sources because of a lack of control on higher order effects. In this paper, we discuss the optimal positioning of octupoles, with respect to the sextupoles present in the lattice, for a local and simultaneous compensation of all fourth order on-momentum phase and amplitude dependent driving terms, using only three families of octupoles. In addition, higher order geometrical terms are also minimized, including among others, second-order tune shift with amplitude. This study is a continuation of past research made on the optimal use of octupoles for the operation of future light sources. The correction method was built on observations made on a simple model, then applied to a realistic low-emittance lattice, designed in the framework of the upgrade of the National Synchrotron Light Source II, to demonstrate its potential.
\end{abstract}

DOI: $10.1103 /$ PhysRevAccelBeams.24.114801

\section{INTRODUCTION}

Finding the right balance between low-emittance lattices and the control of the stronger nonlinear effects, is one of the most challenging task faced by new generations of light sources. The required strong focusing quadrupoles come with the rise of the lattice natural chromaticity. This implies larger sextupole excitations in nonzero dispersion regions to compensate for the negative chromaticity. A technique for simultaneous correction of the chromatic and energyindependent, or geometrical, third-order resonant driving terms (RDTs) generated to the first order by the sextupoles, have been extensively studied and applied in high-energy colliders but also in several upgrades to fourth generation light sources [1-5]. The correction is achieved by constraining the horizontal and vertical betatron phaseadvances $\Delta \mu_{x, y}$ and $\beta_{x, y}$ amplitudes to form a minus identity -I transformation between the sextupole families as detailed in Refs. [6-8].

However, the correction of third-order driving terms is often not sufficient to provide enough dynamic aperture (DA). Large DA, defining the area in the phase space within

\footnotetext{
*fplassard@bnl.gov
}

Published by the American Physical Society under the terms of the Creative Commons Attribution 4.0 International license. Further distribution of this work must maintain attribution to the author(s) and the published article's title, journal citation, and DOI. which the particle motion stays stable, is essential for efficient beam injection. The cross talks between the firstorder sextupole RDTs generate amplitude dependent tune shift (ADTS), fourth (see the Appendix) and higher order phase-dependent terms affecting the overall efficiency of the machine. The remaining higher order RDTs and especially the ADTS, are usually minimized via additional sextupoles in zero-dispersion region, called harmonic sextupoles [9]. Due to the complex mechanism governing second-order perturbation on the beam dynamics, the harmonic sextupoles are optimized by means of numerical tools and high-performance computers to explore and optimize the parameter space [10-13]. A different way of correcting second-order effects of the sextupoles is to use octupole magnets. Octupoles have been studied for their efficient cancellation of linear amplitude detuning terms [14-16]. However, while correcting the linear ADTS, octupoles generate additional nonlinear perturbations that can rapidly limit the machine performance. Notably, phasedependent octupole-like resonances can add up with the ones produced to the second order by the sextupoles [17-19]. The latter are detailed in Appendix. In addition, cross talks among octupole RDTs, produce important higher order terms, contributing to the more chaotic behavior of the particles. These additional unfavorable effects from octupoles have been discussed in past studies in Refs. [15,16]. Simultaneous compensation of those additional high-order effects with octupoles is the main goal of this paper. 
Our nonlinear scheme uses three families of octupoles, powered to control the linear ADTS and for which their location is optimized with respect to the lattice sextupoles, to produce octupole-like RDTs that systematically counteract the ones generated to the second order by the sextupoles with similar amplitudes. In a domino effect, the resulting higher order geometrical RDTs, simulated here up to the sixth order (dodecapole), are also systematically minimized. Dodecapole terms drive second-order ADTS that dominate the tune shift at large excursion and thus the DA. The effectiveness of the scheme has been demonstrated on a simple symmetric and periodic lattice model, alternating focusing and defocusing quadrupoles (FODO), detailed in Sec. II and on a realistic lattice option for the National Synchrotron Light Source II (NSLS-II) upgrade, for which good agreement with the model predictions is observed. This nonlinear correction configuration consistently increase the on-momentum DA by acting on the entire spectrum of the geometrical RDTs, by design and without the intervention of numerical optimization tools (see Sec. III). The impact on chromatic effects and possible use of this scheme features on future light source designs are discussed as well.

\section{FINDING OPTIMAL LOCATION OF OCTUPOLES FOR ON-MOMENTUM CORRECTION}

At injection the beam experience large horizontal deviation from the ideal orbit. The resulting betatron tune shift can bring the particle onto potentially unfavorable resonance conditions. To the first order, the change in transverse tune $\nu_{x, y}$ depends on the amplitude of the particle action $J_{x, y}$, as in Eq. (1):

$$
\begin{aligned}
& \nu_{x}=\nu_{x 0}+\alpha_{x x} J_{x}+\alpha_{x y} J_{y} \quad \text { and } \\
& \nu_{y}=\nu_{y 0}+\alpha_{y y} J_{y}+\alpha_{x y} J_{x} .
\end{aligned}
$$

The linear anharmonicities $\alpha_{x x}, \alpha_{x y}$, and $\alpha_{y y}$, are generated to the second order by the sextupoles as

$$
\begin{aligned}
\alpha_{x x}= & \frac{1}{2 \pi} \frac{\partial^{2} \tilde{H}_{2200}^{(2)}}{\partial J_{x}^{2}}=\sum_{w=1}^{W} \sum_{u=1}^{w-1}\left\{\frac{-1}{64 \pi} b_{3, w} b_{3, u} \beta_{x, w}^{\frac{3}{2}} \beta_{x, u}^{\frac{3}{2}}\right. \\
\times & {\left.\left[3 \sin \left(\phi_{x, u}-\phi_{x, w}\right)+\sin \left(3 \phi_{x, u}-3 \phi_{x, w}\right)\right]\right\} } \\
\alpha_{y y}= & \frac{1}{2 \pi} \frac{\partial^{2} \tilde{H}_{0022}^{(2)}}{\partial J_{y}^{2}}=\sum_{w=1}^{W} \sum_{u=1}^{w-1}\left\{\frac{-1}{64 \pi} b_{3, w} b_{3, u}\right. \\
& \times \sqrt{\beta_{x, w}} \sqrt{\beta_{x, u}} \beta_{y, w} \beta_{y, u}\left[4 \sin \left(\phi_{x, u}-\phi_{x, w}\right)\right. \\
& +\sin \left(\phi_{x, u}-\phi_{x, w}+2 \phi_{y, u}-2 \phi_{y, w}\right) \\
& \left.\left.-\sin \left(\phi_{x, u}-\phi_{x, w}-2 \phi_{y, u}+2 \phi_{y, w}\right)\right]\right\}
\end{aligned}
$$

$$
\begin{aligned}
\alpha_{x y}= & \frac{1}{2 \pi} \frac{\partial^{2} \tilde{H}_{1111}^{(2)}}{\partial J_{x} J_{y}}=\sum_{w=1}^{W} \sum_{u=1}^{w-1}\left\{\frac{1}{32 \pi} b_{3, w} b_{3, u}\right. \\
& \times \sqrt{\beta_{x, w}} \beta_{x, u}^{\frac{3}{2}} \beta_{y, w} \sin \left(\phi_{x, u}-\phi_{x, w}\right) \\
& +\beta_{x, w}^{\frac{3}{2}} \sqrt{\beta_{x, u}} \beta_{y, u} \sin \left(\phi_{x, u}-\phi_{x, w}\right) \\
& -\sqrt{\beta_{x, w}} \sqrt{\beta_{x, u}} \beta_{y, w} \beta_{y, u} \\
& \times\left[\sin \left(\phi_{x, u}-\phi_{x, w}+2 \phi_{y, u}-2 \phi_{y, w}\right)\right. \\
& \left.\left.+\sin \left(-\phi_{x, u}+\phi_{x, w}+2 \phi_{y, u}-2 \phi_{y, w}\right)\right]\right\},
\end{aligned}
$$

where $u, w$ are the index of the position of the sextupoles, $\phi_{x, y}$ is the phase advance, $b_{3}$ is the integrated strength of the sextupoles, and the second-order Hamiltonians $\tilde{H}_{2200}^{(2)}$, $\tilde{H}_{1111}^{(2)}$, and $\tilde{H}_{0022}^{(2)}$ are derived as in Appendix and in Ref. [17]. In the case of low-emittance lattices, where the sextupole strengths are typically strong, the linear tune shift with amplitude can become significant even for small beam transverse offset. At first, we take the same approach for the correction of linear ADTS as studied in Refs. $[6,15,16]$ where three octupole families will act to the first order to cancel them. Their strengths are calculated analytically, by solving the linear system as in Eq. (6):

$$
\overrightarrow{k_{\mathrm{oct}}}=\mathcal{U}_{\mathrm{oct}}^{-1} \cdot \vec{\alpha}
$$

where $\overrightarrow{k_{\text {oct }}}$ is the vector strength of the three octupoles, $\vec{\alpha}$ contains the three direct and cross terms of the linear ADTS as calculated in Eqs. (3), (5), and (4), and $\mathcal{U}_{\text {oct }}$ is the $3 \times 3$ matrix, containing the first-order contribution of the three octupoles, defined as

$$
\mathcal{U}_{\mathrm{oct}}=\frac{1}{8 \pi}\left(\begin{array}{ccc}
\frac{1}{2} \beta_{x \mid \mathrm{oct}_{1}}^{2} & \frac{1}{2} \beta_{x \mid \mathrm{oct}_{2}}^{2} & \frac{1}{2} \beta_{x \mid \mathrm{oct}_{3}}^{2} \\
\frac{1}{2} \beta_{y \mid \mathrm{oct}_{1}}^{2} & \frac{1}{2} \beta_{y \mid \mathrm{oct}_{2}}^{2} & \frac{1}{2} \beta_{y \mid \mathrm{oct}_{3}}^{2} \\
-\beta_{x} \beta_{y \mid \mathrm{oct}_{1}} & -\beta_{x} \beta_{y \mid \mathrm{oct}_{2}} & -\beta_{x} \beta_{y \mid \mathrm{oct}_{3}}
\end{array}\right) .
$$

In order to built a correction method applicable to most modern light source designs, a toy model was developed, simulating the $-\mathcal{I}_{x, y}$ sextupole pairs configuration. A FODO lattice is created and two pairs of sextupoles are inserted at the peaks of $\beta_{x, y}$ and are separated by a horizontal and vertical phase advances $\Delta \mu_{x, y}^{\mathrm{S}}=$ $(2 n+1) \pi$. The betatron amplitude, phases, and magnet strength conditions, form an exact $-\mathcal{I}_{x, y}$ transformation between the sextupole pairs. Three octupoles are then placed close to each other at locations of high $\frac{\beta x}{\beta_{y}}$, high $\frac{\beta y}{\beta_{x}}$, and $\frac{\beta x}{\beta_{y}} \approx 1$, in order to correct, with minimal strength, for $\alpha_{x x}, \alpha_{y y}$, and $\alpha_{x y}$, respectively.

The impact of the octupole triplet location is observed for various different positions in the model, illustrated in 


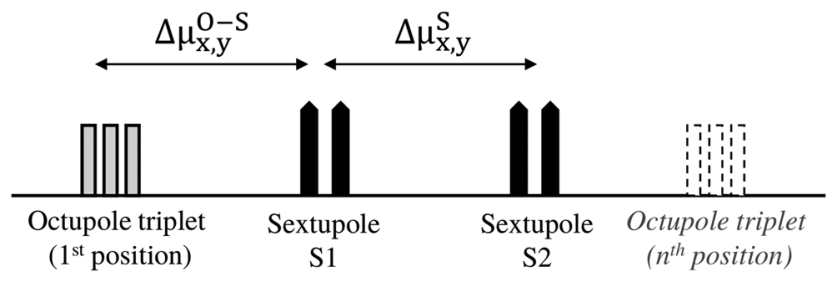

FIG. 1. Schematic of the octupole triplet position scan. $\Delta \mu_{x, y}^{\mathrm{O}-\mathrm{S}}$ is the phase advance between the octupole triplet and the first sextupole. $\Delta \mu_{x, y}^{\mathrm{S}}$ is the phase advance between the sextupoles and is equal to $(2 n+1) \pi$.

Fig. 1, with the same $\beta$-function conditions and thus with the same strength $\overrightarrow{k_{\text {oct }}}$ for an exact cancellation of $\vec{\alpha}$. For each location, all RDTs are recalculated at the end of the optics sequence. This scan shows the impact of octupole triplet positions, when powered for the same correction of linear ADTS, on the amplitude of the RDTs. The scan has been applied for various conditions of $\Delta \mu_{x, y}^{\mathrm{S}}$, e.g., $(\pi, \pi)$, $(3 \pi, 3 \pi)$, and $(3 \pi, \pi)$. The results in Fig. 2 show the sum of all geometrical $s$-dependent Hamiltonian RDTs of order $n=4$ and all dodecapole geometrical RDTs $(n=6)$, computed at each position of the octupole triplet along the FODO lattice using MADX-PTC [20] tracking code. It is clear from the different scans performed, that particular positions of the triplet, with respect to the sextupoles, present significant compensation of these terms. Figure 2 also shows that the level of dodecapole RDTs, that include second-order ADTS, is only reduced at the optimal octupole positions. Similar compensation are observed for decapole RDTs $(n=5)$. The pattern that emerges for every FODO model simulated, and only if $\Delta \mu_{x, y}^{\mathrm{S}}=(2 n+1) \pi$, is that the compensation appears when the octupole triplet is located at
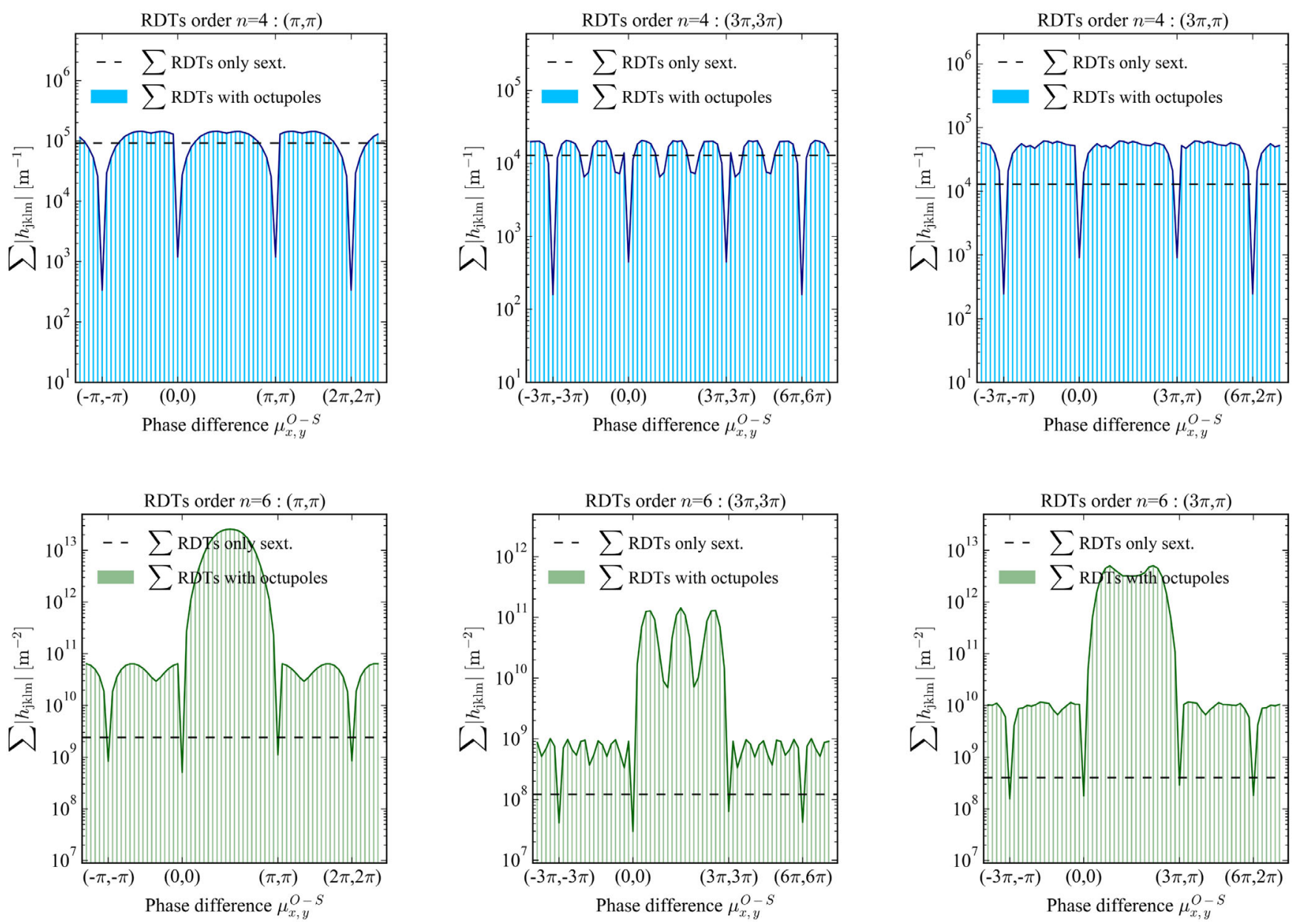

FIG. 2. Top plot: Sum of all fourth order geometrical RDTs (order $n=4$ ) as function of the position of the octupole triplet with respect to the sextupoles. The three RDTs contributing to linear ADTS are not included in the sum as they are fully cancelled at each position; Bottom plot: Sum of all sixth order geometrical RDTs $(n=6)$, including the amplitude dependent terms driving the second order ADTS, as function of the position of the octupole triplet with respect to the sextupoles. From left to right, the scan has been applied for $\Delta \mu_{x, y}^{\mathrm{S}}=(\pi, \pi),(3 \pi, 3 \pi)$, and $(3 \pi, \pi)$. The value of the RDT sum drops drastically at the sextupole location $(m=0)$ and at $\Delta \mu_{x, y}^{\mathrm{O}-\mathrm{S}}=m \times \Delta \mu_{x, y}^{\mathrm{S}}$. The black dashed lines show the sum value of the sextupole contribution only. 


$$
\Delta \mu_{x, y}^{\mathrm{O}-\mathrm{S}}=m \times \Delta \mu_{x, y}^{\mathrm{S}},
$$

where $m$ is an integer. When these conditions are met, all the fourth-order geometrical RDTs generated to the first order by the octupoles $\left(\tilde{H}_{j k l m \mid \text { Oct }}^{(1)}\right)$ and to the second order by the sextupoles $\left(\tilde{H}_{j k l m \mid \operatorname{Sext}}^{(2)}\right)$ always counteract each other with similar amplitudes. This ensure a systematic compensation of all the phase-dependent terms of order $n=4$, simultaneously with the cancellation of the linear ADTS coefficients. In addition, the scheme ensure minimal strength of higher order terms, providing the conditions for a quasi full high order geometrical correction of the system, within one cell.

\section{APPLICATION OF THE CORRECTION SCHEME ON A LOW-EMITTANCE LATTICE}

The design of a light source is often optimized under a large number a constraints, from the storage ring geometry, electro-magnet strengths, optical functions in key regions of the lattice and sometimes phase-advance between sextupoles. Therefore, adding phase constraints between octupoles and sextupoles during the lattice optimization process, as suggested previously, can be troublesome in practice. A simple optimal location for the octupole triplet, in order to meet the high-order geometrical correction discussed in Section II, is in phase with the chromatic sextupoles $(m=0)$. However, the octupoles will be inevitably placed in dispersion region and thus generate additional nonlinear chromatic effects, that need to be restrained. As a proof-of-principle, the octupole scheme has been tested on one of the ultralow emittance lattice options optimized for the upgrade of the NSLS-II. The cell design and optical functions are shown in Fig. 3. This preliminary option provides an equilibrium horizontal emittance of $\epsilon_{x}=25.3 \mathrm{pm}$ at a beam energy of $3 \mathrm{GeV}$, while fitting the tunnel with a circumference of $792 \mathrm{~m}$ and the insertion devices (IDs) source points of the existing

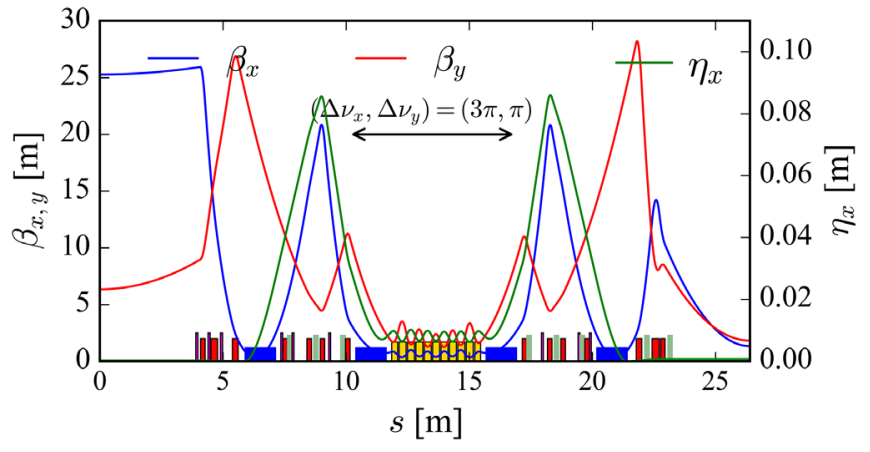

FIG. 3. Twiss functions along one cell of a lattice option for the upgrade of NSLS-II, based on Complex Bend technology. The CB magnet is colored in yellow, the sextupoles in green, and the octupoles in pink.
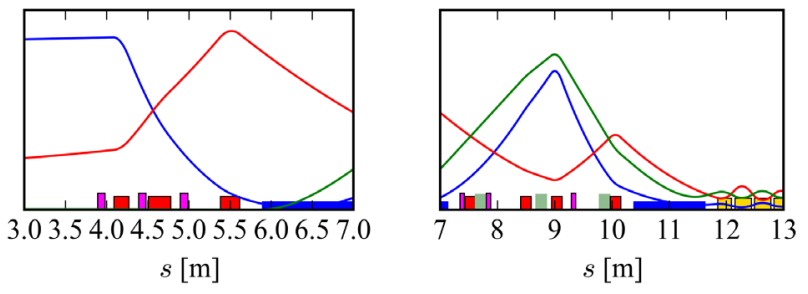

FIG. 4. Left: Position of the octupoles (magenta) in zero dispersion with no phase constraint with respect to the sextupoles; Right: Octupoles location in phase with the chromatic sextupoles.

NSLS-II ring. The ring has a periodicity of 15 , with short and long straight sections for the insertion devices of 7.6 and $6.4 \mathrm{~m}$, respectively. The low emittance is achieved primarily thanks to the use of the novel complex bend (CB) magnet, which consists of a single element with conventional electromagnet dipoles of same field polarity, superposed with strong focusing and defocusing quadrupole field generated by permanent magnets. A detailed technical description of this CB solution is given in Refs. [21,22]. There are two families of chromatic sextupoles powered to correct the natural chromaticity to $(+2,+2)$ units. The three pairs of sextupole (one focusing, two defocusings) are separated by a phase advance of $\left(\Delta \nu_{x}, \Delta \nu_{y}\right)=(3 \pi, \pi)$. The $-\mathcal{I}_{x, y}$ between each sextupole pair, cancels all third order geometrical RDTs $(n=3)$ within one cell.

The three families of octupoles, referred to as oct $_{C}$, are placed in phase with the sextupoles $(m=0)$ for optimal correction. In order to compare the effect of the octupoles location on the nonlinear dynamics, another triplet $\left(\mathrm{oct}_{H}\right)$ is also installed at the edge of the long straight sections, where the phase differences to the sextupoles $\Delta \mu_{x, y}^{\mathrm{O}-\mathrm{S}}$ do not meet the conditions for the simultaneous correction of geometrical high-order effects. Figure 4 shows the location of both triplet location options in the cell. Their integrated strengths are calculated analytically from Eq. (6), to fully cancel the three linear ADTS terms. The obtained vector of strength for the phased triplet oct $\mathrm{C}_{\mathrm{C}}$ is $\overrightarrow{k_{\text {oct }, \mathrm{C}}}=(-2.88 \times$ $\left.10^{3},-8.50 \times 10^{3}, 3.95 \times 10^{3}\right)\left[\mathrm{m}^{-3}\right] \quad$ and $\quad k_{\mathrm{oct}, \mathrm{H}}^{\overrightarrow{\mathrm{O}}}=$ $\left(-3.45 \times 10^{3}, 2.17 \times 10^{2}, 4.37 \times 10^{2}\right)\left[\mathrm{m}^{-3}\right]$ for the oct $_{\mathrm{H}}$ triplet.

\section{A. Nonlinear on-momentum correction}

For each case, the on-momentum phase-dependent fourth-order terms driven to the second order by sextupoles and to the first order by the octupoles within one cell, are computed from the hamiltonian definitions given in Appendix. The sextupole and octupole contributions can be represented individually by a vector in the complex plane. In Fig. 5, their contributions are compared for the two triplet positions. For the unconstrained triplet case $\mathrm{oct}_{\mathrm{H}}$, the strength of most geometrical fourth-order terms is increased due to the presence of the octupoles. On the 


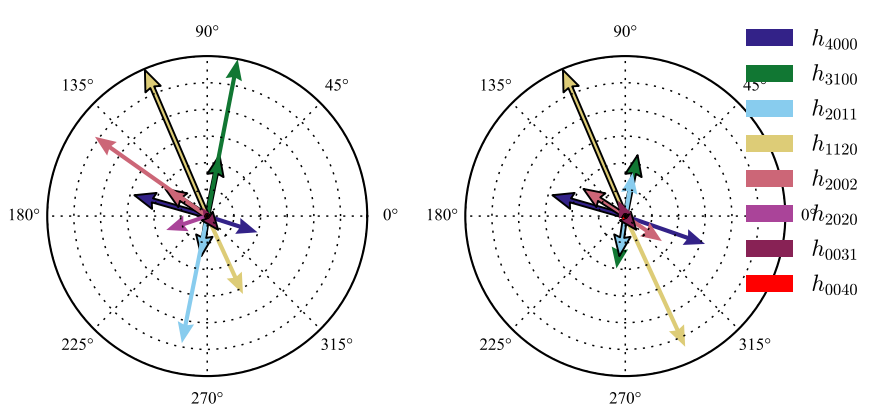

FIG. 5. Vector representation of the 8 fourth-order on-momentum RDTs, calculated from Eqs. (A5), (A6), (A7), (A8), (A9), (A10), (A11), and (A12), generated to second order by the sextupole cross talks (black contoured arrows) and to the first order by the octupoles when powered to fully cancel the linear ADTS. The left plot shows the case of the unphased octupole triplet oct $_{\mathrm{H}}$. The right plot shows the case of the optimal octupoles location, where all fourth RDTs are simultaneously corrected by the oct $_{\mathrm{C}}$ triplet.

contrary, with the triplet oct $_{C}$, installed at the optimal location, their first-order contributions always counteract the ones generated to the second order by the sextupoles with similar amplitudes. This leads to the compensation, within one cell, of all geometrical fourth-order driving terms simultaneously with the full cancellation of the linear ADTS, with only three octupole families for which their strengths are calculated analytically. Table I shows the phase and amplitude dependent RDT values, computed with MADX-PTC after one cell, solely by the lattice chromatic sextupoles and by the octupole triplet oct C $_{\text {. }}$ The sum of these two contributions leads to almost zeroed the amplitude of all fourth-order terms.

The correction of linear ADTS terms can be controlled by applying a weight factor $w_{\text {oct }}$ to the vector strength $\overrightarrow{k_{\text {oct }}}$ as $w_{\text {oct }} \cdot \overrightarrow{k_{\text {oct }}}$. The sum contributions of fourth and higher order on-momentum RDTs as function of $w_{\text {oct }}$ are shown in
Fig. 6. The RDTs amplitude were computed up to the sixth order using MADX-PTC after one turn, for both triplet position options. As the linear ADTS are gradually zeroed, the overall contribution of the octupole-like RDTs increases by $20 \%$ in the case of the oct $_{\mathrm{H}}$ triplet. Furthermore, the sum of second-order amplitude tune shift contributions, driven by the terms $h_{3300}, h_{1122}, h_{2211}$, and $h_{0033}$, increases by more than a factor 2 . As simulated by the FODO model studied in Sec. II, the octupole triplet oct $_{C}$, systematically compensates for all high-order geometrical terms, simultaneously. The sum of the fourth order driving terms and the second order ADTS are decreased by $\approx 90 \%$ and $\approx 50 \%$, respectively. In turns, the on-momentum DA area is gradually enlarged with $w_{\text {oct,C }}$, up to a factor 7 .

These results show the importance of the localization of octupoles with respect to the lattice sextupoles. The performance limitations of using octupoles dedicated to the correction of linear ADTS, have been pointed out in Refs. [15] and [16]. Notably, the rise of second-order ADTS, while correcting linear tune shift, set a strong limitation on the DA improvement. Here, we addressed these issues by showing that at the optimal location, three octupoles will act positively on the entire spectrum of onmomentum resonances. The relevance of reducing nonlinear tune shift is particularly high for light sources using the top-off injection system, like NSLS-II and its upgrade, where the beam must remain stable beyond $5 \mathrm{~mm}$ offset in the horizontal plane. Figure 7 shows the tune shift with horizontal amplitude for three cases: without any octupoles $w_{\text {oct }}=0 \%$, using only oct $\mathrm{H}_{\mathrm{H}}$ triplet and using oct $\mathrm{C}$. The tune shift generated solely by higher order sextupole perturbations, rapidly move the tune toward half or integer resonances with $\Delta x \leq \pm 2.5 \mathrm{~mm}$. When fully correcting linear ADTS with oct $_{\mathrm{H}}$ triplet, the nonlinear effects will dominate the tune shift at larger amplitudes and here the integer resonances is crossed for $\Delta x \geq \pm 4 \mathrm{~mm}$. Now, for the optimal octupole position oct $C$, the linear and nonlinear

TABLE I. Table of the fourth-order RDTs generated to the second order by the chromatic sextupoles and to the first order by the octupole triplet Oct $_{\mathrm{C}}$ only, after one cell. With Oct $_{\mathrm{C}}$, phase and amplitude resonances are simultaneously and systematically compensated.

\begin{tabular}{lcc}
\hline \hline RDTs $n=4$ & From sextupoles (Second order) & ${\text { From triplet } \text { Oct }_{C} \text { (First order) }}$ \\
\hline$\alpha_{x x}$ & 45772 & -45816 \\
$\alpha_{y y}$ & 6516 & -6518 \\
$\alpha_{x y}$ & -34334 & 34326 \\
$\tilde{H}_{4000}=\tilde{H}_{0400}^{*}$ & $-10784+i 260$ & $10441-i 289$ \\
$\tilde{H}_{3100}=\tilde{H}_{1300}^{*}$ & $128+i 11553$ & $-156-i 11225$ \\
$\tilde{H}_{2011}=\tilde{H}_{0211}^{*}$ & $-117-i 10423$ & $82+i 12603$ \\
$\tilde{H}_{1120}=\tilde{H}_{1102}^{*}$ & $1329+i 54095$ & $-628-i 51242$ \\
$\tilde{H}_{2002}=\tilde{H}_{0220}^{*}$ & $-14293+i 184$ & $13247-i 71$ \\
$\tilde{H}_{2020}=\tilde{H}_{0202}^{*}$ & $-1940+i 75$ & $1146-i 31$ \\
$\tilde{H}_{0031}=\tilde{H}_{0013}^{*}$ & $-98-i 4102$ & $45+i 6200$ \\
$\tilde{H}_{0040}=\tilde{H}_{0004}^{*}$ & $-216+i 12$ & $152-i 22$ \\
\hline \hline
\end{tabular}



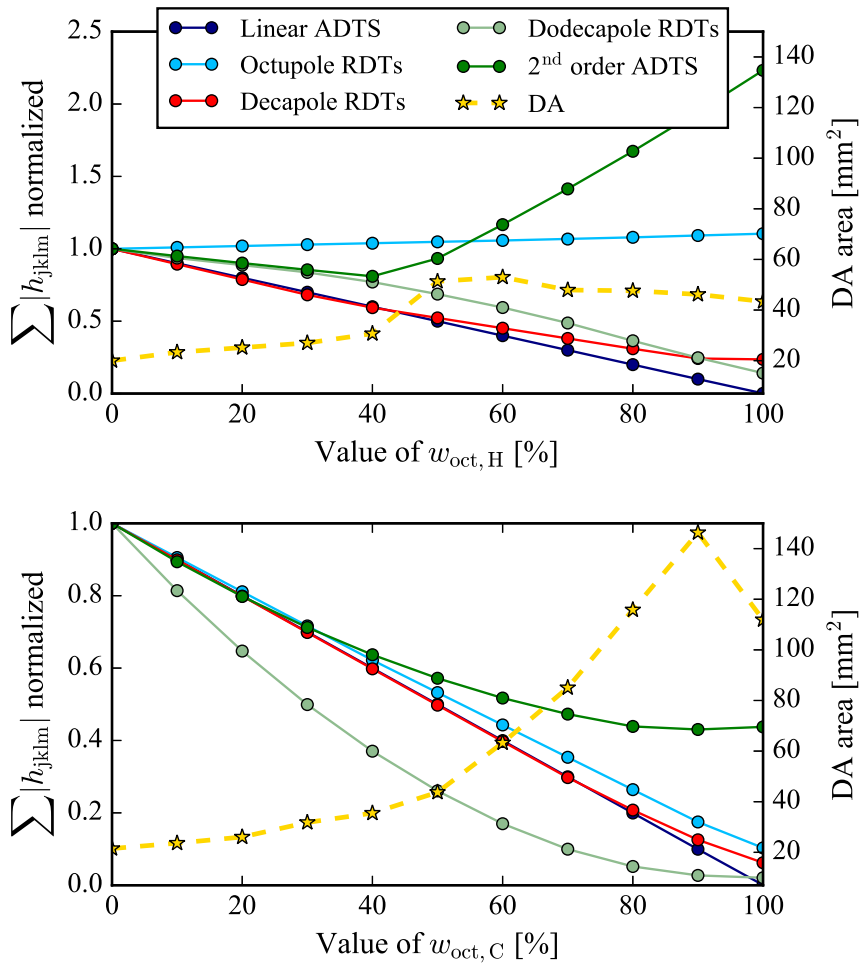

FIG. 6. Sum of the geometrical RDT amplitudes computed with MADX-PTC [20] after one turn for each the fourth-, fifth-, and sixth-order contributions, as function of the weight factor $w_{\text {oct }}$. The values are normalized to the sum contributions of the sextupoles only. The phase and amplitude dependent terms are summed separately in order to distinguish the contribution of the linear and second-order ADTS. The on-momentum DA area is computed after 1024 turns using the ELEGANT [23] tracking code. The top plot shows the case using oct $_{\mathrm{H}}$ triplet. The bottom plots shows the case using oct ${ }_{\mathrm{C}}$ triplet where the contribution of all phase and amplitude dependent terms simultaneously decrease with $w_{\text {oct }}$.

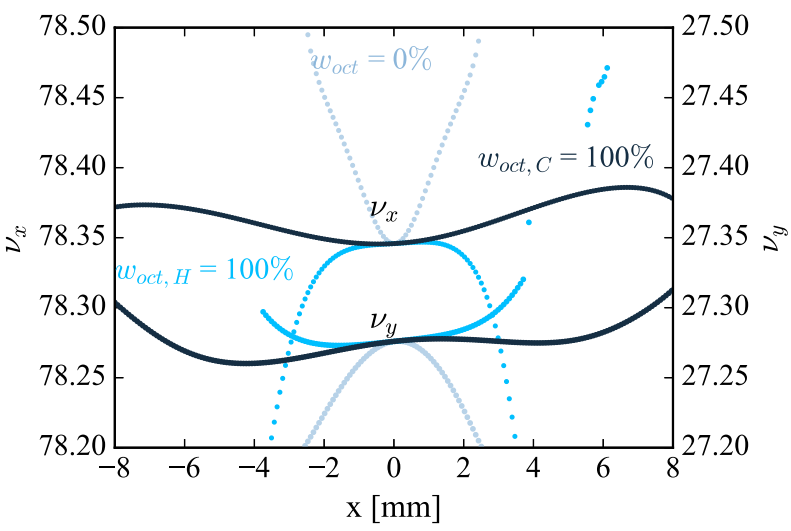

FIG. 7. Tune shift with horizontal amplitude in the case with only sextupoles $\left(w_{\mathrm{oct}}=0 \%\right)$, with oct $_{\mathrm{H}}$ arbitrarily positioned with respect to sextupoles, powered to cancel linear tune shift $\left(w_{\text {oct }, \mathrm{H}}=100 \%\right)$ and in the case with the triplet at the optimal position oct C $_{\text {with }} w_{\text {oct, }}=100 \%$. tune shift are minimized together, leading to a very weak tune shift even at large transverse offset. The half or integer resonances are crossed only for $\Delta x$ beyond $\pm 12 \mathrm{~mm}$. The nonlinear effects are minimized in both the horizontal and vertical planes. Figure 8 shows how the optimal octupole triplet oct $_{\mathrm{C}}$ progressively shrink the tune footprint, allowing for a much larger DA in the horizontal and vertical directions. This tune confinement approach, together with the strength reduction of most geometrical RDTs, enlarge the DA while making the lattice more robust to machine imperfections.

The effect of simultaneous high-order correction has been observed consistently for many different types of low emittance lattices optimized with chromatic sextupole pairs separated by a $-\mathcal{I}_{x, y}$ matrix. This nonlinear correction method offers multiple advantages. While the sextupole scheme ensures the self-compensation of third order geometrical RDTs, the octupole triplet will extend the local on-momentum correction to higher order, by-design, with only three families of octupoles. There is no blind optimization, the compensated nonlinear driving terms correlated to the DA improvement are known and can be tuned accordingly with one knob $w_{\text {oct.C }}$. The systematic tune footprint confinement, even at large excursions, allows to obtain sufficient DA improvement almost independently of the tune working point (WP). Consequently, this gives more degree of freedom on the choice of WP for tailoring the lattice off-momentum dynamics. The latter is of prime importance as the triplet is located near the chromatic sextupoles, in nonzero dispersion region, and will inevitably impact the machine momentum acceptance.

\section{B. Nonlinear chromatic considerations}

Touschek effect can drive particles to large momentum offset $\delta_{p}$, shifting their tunes onto potentially harmful resonances, and ultimately reduce the beam lifetime. Nonlinear chromatic effects set a limit to the achievable momentum aperture of the lattice. The contribution of octupoles to second-order chromaticity can be calculated as

$$
\frac{\partial^{2} \nu_{x, y}}{\partial \delta_{p}^{2}}= \pm \frac{1}{8 \pi} \oint \beta_{x, y}(s) k_{\mathrm{oct}}(s) \eta_{x}^{2}(s) \mathrm{d} s,
$$

where $k_{\text {oct }}$ is the normalized octupole strength and $\eta_{x}$ is the horizontal dispersion function. In most cases, powering the octupole triplet in nonzero dispersion region, with the intent to fully correct high-order geometrical effects, as proposed by our scheme, comes at the expense of increasing second order chromatic tune shift. Hence, one needs to balance between those competing effects to provide both good injection efficiency and beam lifetime. In the present lattice study case, the nonlinear chromaticity was minimized by design during linear optimization. The impact of

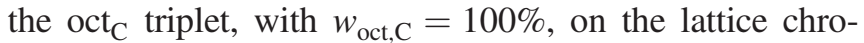
matic tune shift is shown in Fig. 9. In this situation, the 

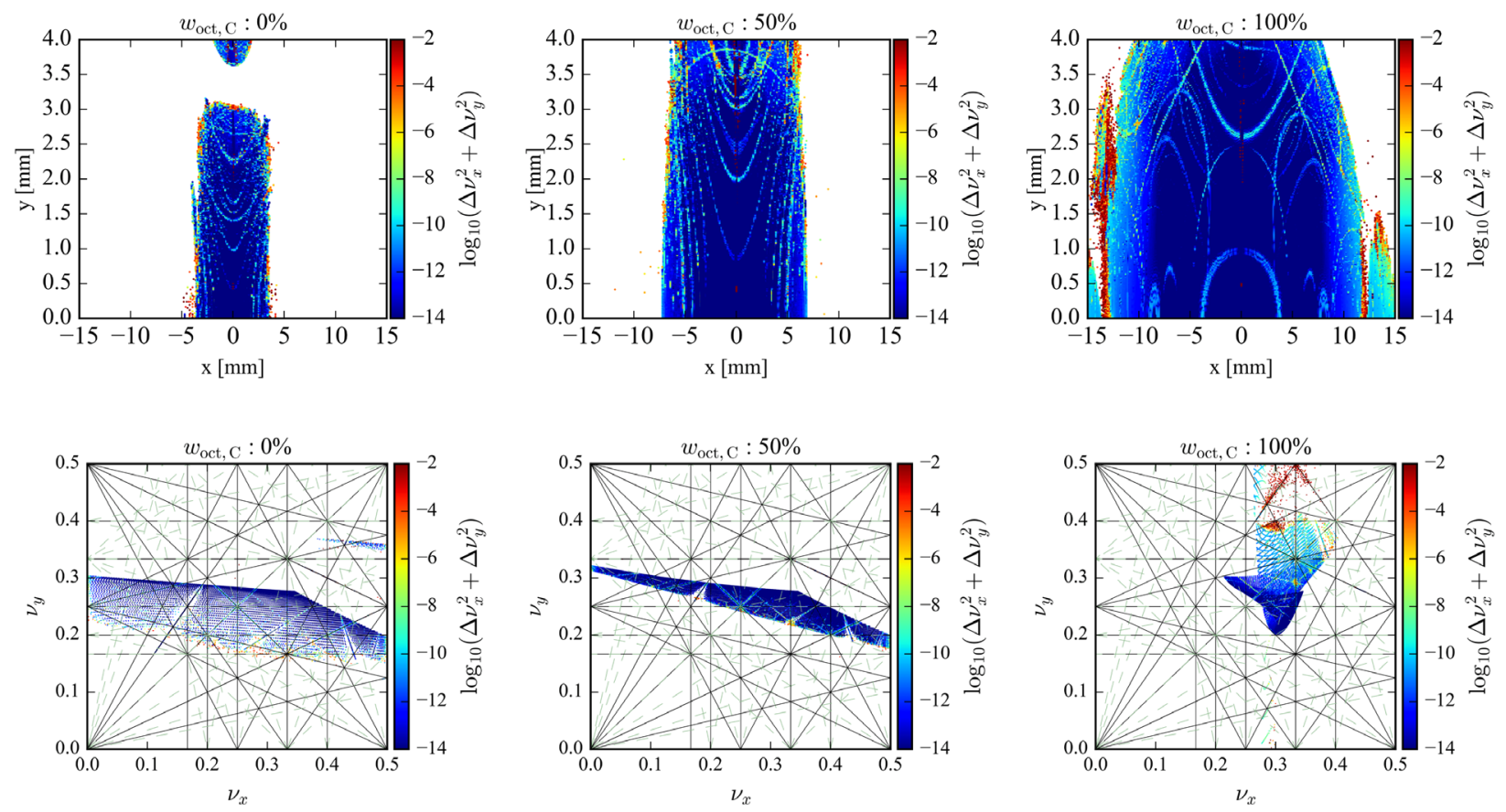

FIG. 8. On-momentum dynamic aperture increase with $w_{\text {oct, }}$, tracked at the injection point for 1024 turns. As the strength of the optimal triplet oct $_{\mathrm{C}}$ increase, the tune footprint is confined into a smaller area in the tune space, making the particles less likely to cross potentially harmful resonances during injection.

reduction of momentum acceptance (MA) is too large for viable machine operation. The MA can be improved by optimizing the peak of $\beta_{x, y}$ and $\eta_{x}$ functions at the dispersion bumps in order to reduce the chromatic impact of the octupoles. There is more flexibility to reduce the dispersion level at the sextupoles, within their strength limit, as the octupole triplet will suppress by design most of the on-momentum perturbations. One can also optimize the WP in order to move further away from half-integer and integer resonances. Ideally, the design would have the octupole triplet located at a phase separation, with respect to the sextupoles, as given in Eq. (8) with $m \neq 0$, in nondispersion region. In this case, high-order geometrical correction could be achieved without acting on the lattice

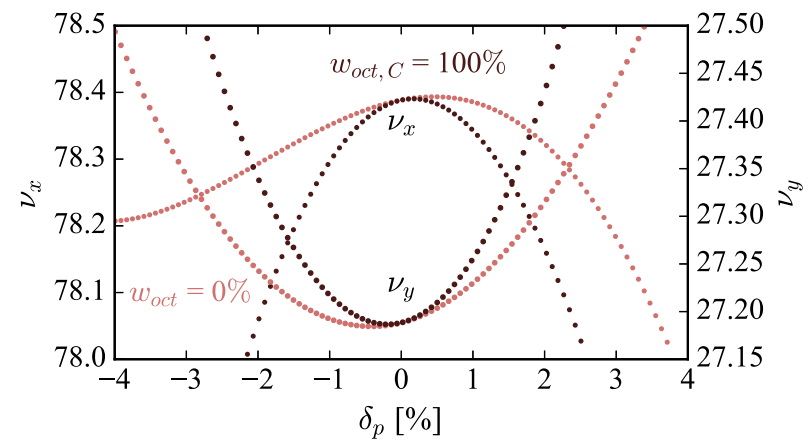

FIG. 9. Comparison of the horizontal and vertical chromatic tune shift, without octupoles and with the oct ${ }_{C}$ triplet fully powered. nonlinear chromaticity. However, the constraints imposed on the NSLS-II upgrade design prevent from efficiently set these phase condition between the sextupoles and the octupoles. Another option, applied here, was to use both oct $_{\mathrm{C}}$ and oct $\mathrm{H}_{\mathrm{H}}$ triplets to limit the increase of second-order chromaticity from oct $\mathrm{C}_{\mathrm{C}}$ while keeping full control of the linear ADTS. The use of the two knobs $w_{\text {oct,C }}$ and $w_{\text {oct, } \mathrm{H}}$ allows a straightforward trade-off between the correction of geometrical and chromatic effects. By simply distributing the weights as $\left(w_{\text {oct, } \mathrm{C}}, w_{\text {oct. } \mathrm{H}}\right)=(70 \%, 30 \%)$, the onmomentum DA is still well improved while keeping the MA within the desired range. The resulting DA and the MA are shown in Figs. 10 and 11, respectively. The momentum

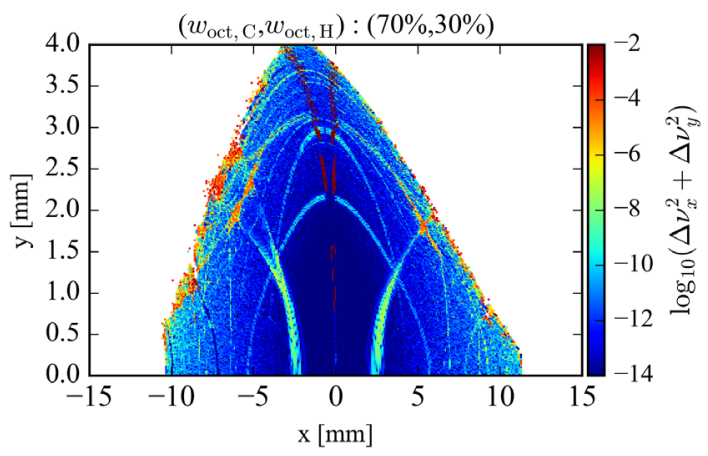

FIG. 10. On-momentum DA using both triplets oct $\mathrm{C}_{\mathrm{C}}$ and oct $_{\mathrm{H}}$ to cancel linear amplitude detuning with $\left(w_{\text {oct, } \mathrm{C}}, w_{\text {oct, } \mathrm{H}}\right)=$ $(70 \%, 30 \%)$, in order to relax their impact on nonlinear chromaticity. 


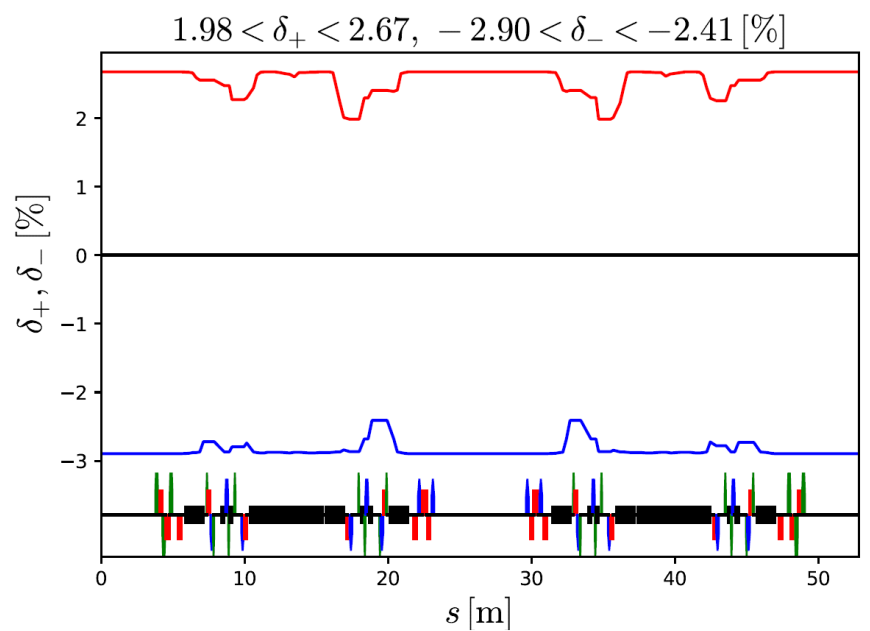

FIG. 11. 6D Momentum acceptance for the octupole setup $\left(w_{\text {oct,C }}, w_{\text {oct.H }}\right)=(70 \%, 30 \%)$, tracked along one super-cell for 2048 turns. An rf cavity was turned on with harmonic number of 1320 and rf bucket height of $2.73 \%$. Radiation loss was included.

aperture was searched by tracking particles for 2048 turns. An rf cavity was turned on with harmonic number of 1320 and $\mathrm{rf}$ bucket height of $2.73 \%$ (rf voltage of $0.543 \mathrm{MV}$; automatically determined from the nonlinear chromaticity aperture data based on integer/half-integer resonance crossing) at the beam energy of $3 \mathrm{GeV}$. Radiation loss was included. The beam lifetime calculated at full coupling, is around $2 \mathrm{~h}$. The large on-momentum DA margin, which is twice the $\pm 5 \mathrm{~mm}$ horizontal DA target, gives room to further increase the beam lifetime. The optimal position of the octupole triplet, handling at once the geometrical effects, gives a powerful leverage to the designer to easily optimize the machine performance up to an operational level, even for ultralow emittance lattices. While the impact of realistic beamline imperfections has not been included in the performance evaluation, the tune confinement approach adopted here, ensure the robustness of the machine in operational conditions.

\section{CONCLUSIONS}

This study gives a recipe for the location of octupole families for the overall compensation of nonlinear geometrical effects. Combining the technique of correcting linear ADTS with three octupoles, together with their optimal positioning with respect to the sextupoles, will provide a simultaneous correction of phase and amplitude-dependent fourth and higher order resonances. Under the appropriate conditions, the fourth-order RDTs generated to the first order by the octupoles systematically counteract the ones generated to the second order by the sextupoles with similar amplitudes. Consequently, higher order perturbations are simultaneously minimized, including second-order ADTS, leading to a considerable increase of on-momentum DA.
This is achieved with only three octupole families, controlling most of the on-momentum RDT spectrum.

This nonlinear correction method has been demonstrated on a simplified FODO model and on a realistic low emittance design, optimized in the framework of the NSLS-II upgrade. It has been tested on a variety of types of multibend achromat light source lattices, featuring the $-\mathcal{I}_{x, y}$ transformer between the sextupole pairs, with excellent consistency. This octupole scheme is a high-order complement to the $-\mathcal{I}_{x, y}$ transformation between chromatic sextupoles. Despite that the octupole triplet does not target the correction of nonlinear off-momentum effects, it was shown that it gives enough flexibility for balancing between geometrical and chromatic corrections in order to reach the required performance. Future lattices could be designed, based on this nonlinear scheme, with the octupole triplet installed in nondispersion region and therefore without degrading the chromatic condition. Finally, this method offers a fast and simple way for the nonlinear optimization of future high-brightness light sources, by acting on a known set of parameters.

\section{ACKNOWLEDGMENTS}

This research used resources of the National Synchrotron Light Source II, a U.S. Department of Energy (DOE) Office of Science User Facility operated for the DOE Office of Science by Brookhaven National Laboratory under Contract No. DE-SC0012704.

\section{APPENDIX: GEOMETRICAL FIRST- AND SECOND-ORDER DRIVING TERMS FROM OCTUPOLES AND SEXTUPOLES}

This section provides the explicit expressions of the firstorder geometrical driving terms generated by the octupoles and the second-order terms arising from the cross talks of thin sextupoles, that were used for the analytical calculation of fourth-order RDTs discussed in Sec. II. The following expressions are a revision of the explicit formulas given in Ref. [19]. These second-order Hamiltonians do not include skew sextupole field components.

The Hamiltonian operators in the Courant-Snyder coordinates are noted $\tilde{H}=\tilde{H}\left(J_{x}, J_{y}, \Phi_{x}, \Phi_{y}\right)$ with $J_{x, y}, \Phi_{x, y}$ the linear invariant and betatron phases, respectively. The operator can be decomposed in first- and second-order terms as

$\tilde{H}=\tilde{H}^{(1)}+\tilde{H}^{(2)}=\sum_{w=1}^{W} \tilde{H}_{w}+\frac{1}{2} \sum_{w=1}^{W} \sum_{u=1}^{w-1}\left[\tilde{H}_{u}, \tilde{H}_{w}\right]$

where $w$ and $u$ are the indices of the multipole locations. By deriving each term from the general definition given in Appendix A of Ref. [24], one obtain the explicit expression for the 11 amplitude and phase-dependent octupole-like driving terms, and their corresponding complex conjugate, 
driving the three linear ADTS as well as the $4 \nu_{x}, 2 \nu_{x}, 4 \nu_{y}, 2 \nu_{y}, 2 \nu_{x}+2 \nu_{y}$, and $2 \nu_{x}-2 \nu_{y}$ resonances. The following equations compute the sum of the driving terms generated to the first order by the octupoles $\tilde{H}_{j k l m}^{(1)}$ and to the second order by the sextupoles $\tilde{H}_{j k l m}^{(2)}$ :

$$
\begin{aligned}
& \tilde{H}_{2200}^{(1)}+\tilde{H}_{2200}^{(2)}=\sum_{w=1}^{W}-\frac{b_{4, w}}{64} \beta_{x, w}^{2} h_{x,+}^{2} h_{x,-}^{2}+i \sum_{w=1}^{W} \sum_{u=1}^{w-1}\left\{\frac { b _ { 3 , w } b _ { 3 , u } } { 2 5 6 } \beta _ { x , w } ^ { \frac { 3 } { 2 } } \beta _ { x , u } ^ { \frac { 3 } { 2 } } \left(-3 e^{i\left[\Delta \phi_{x, u}-\Delta \phi_{x, w}\right]}+3 e^{i\left[-\Delta \phi_{x, u}+\Delta \phi_{x, w}\right]}\right.\right. \\
& \left.\left.-e^{i\left[3 \Delta \phi_{x, u}-3 \Delta \phi_{x, w}\right]}+e^{i\left[-3 \Delta \phi_{x, u}+3 \Delta \phi_{x, w}\right]}\right)\right\} h_{x,+}^{2} h_{x,-}^{2}, \\
& \tilde{H}_{1111}^{(1)}+\tilde{H}_{1111}^{(2)}=\sum_{w=1}^{W}-\frac{b_{4, w}}{16} \beta_{x, w} \beta_{y, w} h_{x,+} h_{x,-} h_{y,+} h_{y,-}+i \sum_{w=1}^{W} \sum_{u=1}^{w-1}\left\{\frac { b _ { 3 , w } b _ { 3 , u } } { 6 4 } \left[\sqrt{\beta_{x, w}} \beta_{x, u}^{\frac{3}{2}} \beta_{y, w}\left(e^{i\left[\Delta \phi_{x, u}-\Delta \phi_{x, w}\right]}-e^{i\left[-\Delta \phi_{x, u}+\Delta \phi_{x, w}\right]}\right)\right.\right. \\
& +\beta_{x, w}^{\frac{3}{2}} \sqrt{\beta_{x, u}} \beta_{y, u}\left(e^{i\left[\Delta \phi_{x, u}-\Delta \phi_{x, w}\right]}-e^{i\left[-\Delta \phi_{x, u}+\Delta \phi_{x, w}\right]}\right) \\
& +\sqrt{\beta_{x, w}} \sqrt{\beta_{x, u}} \beta_{y, w} \beta_{y, u}\left(-e^{i\left[\Delta \phi_{x, u}-\Delta \phi_{x, w}+2 \Delta \phi_{y, u}-2 \Delta \phi_{y, w}\right]}-e^{i\left[-\Delta \phi_{x, u}+\Delta \phi_{x, w}+2 \Delta \phi_{y, u}-2 \Delta \phi_{y, w}\right]}\right. \\
& \left.\left.\left.+e^{i\left[\Delta \phi_{x, u}-\Delta \phi_{x, w}-2 \Delta \phi_{y, u}+2 \Delta \phi_{y, w}\right]}+e^{i\left[-\Delta \phi_{x, u}+\Delta \phi_{x, w}-2 \Delta \phi_{y, u}+2 \Delta \phi_{y, w}\right]}\right)\right]\right\} h_{x,+} h_{x,-} h_{y,+} h_{y,-}, \\
& \tilde{H}_{0022}^{(1)}+\tilde{H}_{0022}^{(2)}=\sum_{w=1}^{W}-\frac{b_{4, w}}{64} \beta_{y, w}^{2} h_{y,+}^{2} h_{y,-}^{2}+i \sum_{w=1}^{W} \sum_{u=1}^{w-1}\left\{\frac { b _ { 3 , w } b _ { 3 , u } } { 2 5 6 } \sqrt { \beta _ { x , w } } \sqrt { \beta _ { x , u } } \beta _ { y , w } \beta _ { y , u } \left(-4 e^{i\left[\Delta \phi_{x, u}-\Delta \phi_{x, w}\right]}\right.\right. \\
& +4 e^{i\left[-\Delta \phi_{x, u}+\Delta \phi_{x, w}\right]}-e^{i\left[\Delta \phi_{x, u}-\Delta \phi_{x, w}+2 \Delta \phi_{y, u}-2 \Delta \phi_{y, w}\right]}+e^{i\left[-\Delta \phi_{x, u}+\Delta \phi_{x, w}+2 \Delta \phi_{y, u}-2 \Delta \phi_{y, w}\right]} \\
& \left.-e^{i\left[\Delta \phi_{x, u}-\Delta \phi_{x, w}-2 \Delta \phi_{y, u}+2 \Delta \phi_{y, w}\right]}+e^{i\left[-\Delta \phi_{x, u}+\Delta \phi_{x, w}-2 \Delta \phi_{y, u}+2 \Delta \phi_{y, w}\right]}\right) h_{y,+}^{2} h_{y,-}^{2}, \\
& \tilde{H}_{4000}^{(1)}+\tilde{H}_{4000}^{(2)}=\sum_{w=1}^{W}-\frac{b_{4, w}}{384} \beta_{x, w}^{2} e^{i\left[4 \phi_{x, w}\right]} h_{x,+}^{4}+i \sum_{w=1}^{W} \sum_{u=1}^{w-1}\left\{\frac{b_{3, w} b_{3, u}}{256} \beta_{x, w}^{\frac{3}{2}} \beta_{x, u}^{\frac{3}{2}} \times\left(e^{i\left[\phi_{x, u}+3 \phi_{x, w}\right]}-e^{i\left[3 \phi_{x, u}+\phi_{x, w}\right]}\right)\right\} h_{x,+}^{4}, \\
& \tilde{H}_{3100}^{(1)}+\tilde{H}_{3100}^{(2)}=\sum_{w=1}^{W}-\frac{b_{4, w}}{96} \beta_{x, w}^{2} e^{i\left[2 \phi_{x, w}\right]} h_{x,+}^{3} h_{x,-}+i \sum_{w=1}^{W} \sum_{u=1}^{w-1}\left\{\frac{b_{3, w} b_{3, u}}{128} \beta_{x, w}^{\frac{3}{2}} \beta_{x, u}^{\frac{3}{2}} \times\left(e^{i\left[-\phi_{x, u}+3 \phi_{x, w}\right]}-e^{i\left[3 \phi_{x, u}-\phi_{x, w}\right]}\right)\right\} h_{x,+}^{3} h_{x,-}, \\
& \tilde{H}_{2011}^{(1)}+\tilde{H}_{2011}^{(2)}=\sum_{w=1}^{W}-\frac{b_{4, w}}{32} \beta_{x, w} \beta_{y, w} e^{i\left[2 \phi_{x, w}\right]} h_{x,+}^{2} h_{y,+} h_{y,-}+i \sum_{w=1}^{W} \sum_{u=1}^{w-1}\left\{\frac { b _ { 3 , w } b _ { 3 , u } } { 1 2 8 } \left[\sqrt{\beta_{x, w}} \beta_{x, u}^{\frac{3}{2}} \beta_{y, w} \times\left(e^{i\left[3 \phi_{x, u}-\phi_{x, w}\right]}-e^{i\left[\phi_{x, u}+\phi_{x, w}\right]}\right)\right.\right. \\
& +\beta_{x, w}^{\frac{3}{2}} \sqrt{\beta_{x, u}} \beta_{y, u}\left(e^{i\left[\phi_{x, u}+\phi_{x, w}\right]}-e^{i\left[-\phi_{x, u}+3 \phi_{x, w}\right]}\right)+2 \sqrt{\beta_{x, w}} \sqrt{\beta_{x, u}} \beta_{y, w} \beta_{y, u} \\
& \left.\left.\times\left(e^{i\left[\phi_{x, u}+\phi_{x, w}-2 \phi_{y, u}+2 \phi_{y, w}\right]}-e^{i\left[\phi_{x, u}+\phi_{x, w}+2 \phi_{y, u}-2 \phi_{y, w}\right]}\right)\right]\right\} h_{x,+}^{2} h_{y,+} h_{y,-}, \\
& \tilde{H}_{1120}^{(1)}+\tilde{H}_{1120}^{(2)}=\sum_{w=1}^{W}-\frac{b_{4, w}}{32} \beta_{x, w} \beta_{y, w} e^{i\left[2 \phi_{y, w}\right]} h_{x,+} h_{x,-} h_{y,+}^{2}+i \sum_{w=1}^{W} \sum_{u=1}^{w-1}\left\{\frac { b _ { 3 , w } b _ { 3 , u } } { 1 2 8 } \left[\sqrt{\beta_{x, w}} \beta_{x, u}^{\frac{3}{2}} \beta_{y, w}\right.\right. \\
& \times\left(e^{i\left[\phi_{x, u}-\phi_{x, w}+2 \phi_{y, w}\right]}-e^{i\left[-\phi_{x, u}+\phi_{x, w}+2 \phi_{y, w}\right]}\right)+\beta_{x, w}^{\frac{3}{2}} \sqrt{\beta_{x, u}} \beta_{y, u}\left(e^{i\left[\phi_{x, u}-\phi_{x, w}+2 \phi_{y, u}\right]}-e^{i\left[-\phi_{x, u}+\phi_{x, w}+2 \phi_{y, u}\right]}\right) \\
& +2 \sqrt{\beta_{x, w}} \sqrt{\beta_{x, u}} \beta_{y, w} \beta_{y, u}\left(-e^{i\left[\phi_{x, u}-\phi_{x, w}+2 \phi_{y, u}\right]}-e^{i\left[-\phi_{x, u}+\phi_{x, w}+2 \phi_{y, u}\right]}+e^{i\left[\phi_{x, u}-\phi_{x, w}+2 \phi_{y, w}\right]}\right. \\
& \left.\left.\left.+e^{i\left[-\phi_{x, u}+\phi_{x, w}+2 \phi_{y, w}\right]}\right)\right]\right\} h_{x,+} h_{x,-} h_{y,+}^{2} \text {, }
\end{aligned}
$$




$$
\begin{aligned}
& \tilde{H}_{2002}^{(1)}+\tilde{H}_{2002}^{(2)}=\sum_{w=1}^{W}-\frac{b_{4, w}}{64} \beta_{x, w} \beta_{y, w} e^{i\left[2 \phi_{x, w}-2 \phi_{y, w}\right]} h_{x,+}^{2} h_{y,-}^{2} \\
& +i \sum_{w=1}^{W} \sum_{u=1}^{w-1}\left\{\frac { b _ { 3 , w } b _ { 3 , u } } { 2 5 6 } \left[\sqrt{\beta_{x, w}} \beta_{x, u}^{\frac{3}{2}} \beta_{y, w}\left(e^{i\left[3 \phi_{x, u}-\phi_{x, w}-2 \phi_{y, w}\right]}-e^{i\left[\phi_{x, u}+\phi_{x, w}-2 \phi_{y, w}\right]}\right)\right.\right. \\
& +\beta_{x, w}^{\frac{3}{2}} \sqrt{\beta_{x, u}} \beta_{y, u}\left(e^{i\left[\phi_{x, u}+\phi_{x, w}-2 \phi_{y, u}\right]}-e^{i\left[-\phi_{x, u}+3 \phi_{x, w}-2 \phi_{y, u}\right]}\right) \\
& \left.\left.+4 \sqrt{\beta_{x, w}} \sqrt{\beta_{x, u}} \beta_{y, w} \beta_{y, u}\left(e^{i\left[\phi_{x, u}+\phi_{x, w}-2 \phi_{y, u}\right]}-e^{i\left[\phi_{x, u}+\phi_{x, w}-2 \phi_{y, w}\right]}\right)\right]\right\} h_{x,+}^{2} h_{y,-}^{2}, \\
& \tilde{H}_{2020}^{(1)}+\tilde{H}_{2020}^{(2)}=\sum_{w=1}^{W}-\frac{b_{4, w}}{64} \beta_{x, w} \beta_{y, w} e^{i\left[2 \phi_{x, w}+2 \phi_{y, w}\right]} h_{x,+}^{2} h_{y,+}^{2} \\
& +i \sum_{w=1}^{W} \sum_{u=1}^{w-1}\left\{\frac { b _ { 3 , w } b _ { 3 , u } } { 2 5 6 } \left[\sqrt{\beta_{x, w}} \beta_{x, u}^{\frac{3}{2}} \beta_{y, w}\left(e^{i\left[3 \phi_{x, u}-\phi_{x, w}+2 \phi_{y, w}\right]}-e^{i\left[\phi_{x, u}+\phi_{x, w}+2 \phi_{y, w}\right]}\right)\right.\right. \\
& +\beta_{x, w}^{\frac{3}{2}} \sqrt{\beta_{x, u}} \beta_{y, u}\left(e^{i\left[\phi_{x, u}+\phi_{x, w}+2 \phi_{y, u}\right]}-e^{i\left[-\phi_{x, u}+3 \phi_{x, w}+2 \phi_{y, u}\right]}\right) \\
& \left.\left.+4 \sqrt{\beta_{x, w}} \sqrt{\beta_{x, u}} \beta_{y, w} \beta_{y, u}\left(-e^{i\left[\phi_{x, u}+\phi_{x, w}+2 \phi_{y, u}\right]}+e^{i\left[\phi_{x, u}+\phi_{x, w}+2 \phi_{y, w}\right]}\right)\right]\right\} h_{x,+}^{2} h_{y,+}^{2}, \\
& \tilde{H}_{0031}^{(1)}+\tilde{H}_{0031}^{(2)}=\sum_{w=1}^{W}-\frac{b_{4, w}}{96} \beta_{y, w}^{2} e^{i\left[2 \phi_{y, w}\right]} h_{y,+}^{3} h_{y,-}+i \sum_{w=1}^{W} \sum_{u=1}^{w-1}\left\{\frac { b _ { 3 , w } b _ { 3 , u } } { 1 2 8 } \left[\sqrt { \beta _ { x , w } } \sqrt { \beta _ { x , u } } \beta _ { y , w } \beta _ { y , u } \left(-e^{i\left[\phi_{x, u}-\phi_{x, w}+2 \phi_{y, u}\right]}\right.\right.\right. \\
& \left.\left.\left.+e^{i\left[-\phi_{x, u}+\phi_{x, w}+2 \phi_{y, u}\right]}-e^{i\left[\phi_{x, u}-\phi_{x, w}+2 \phi_{y, w}\right]}+e^{i\left[-\phi_{x, u}+\phi_{x, w}+2 \phi_{y, w}\right]}\right)\right]\right\} h_{y,+}^{3} h_{y,-}, \\
& \tilde{H}_{0040}^{(2)}=\sum_{w=1}^{W}-\frac{b_{4, w}}{384} \beta_{y, w}^{2} e^{i\left[4 \phi_{y, w}\right]} h_{y,+}^{4}+i \sum_{w=1}^{W} \sum_{u=1}^{w-1}\left\{\frac { b _ { 3 , w } b _ { 3 , u } } { 2 5 6 } \left[\sqrt { \beta _ { x , w } } \sqrt { \beta _ { x , u } } \beta _ { y , w } \beta _ { y , u } \left(-e^{i\left[\phi_{x, u}-\phi_{x, w}+2 \phi_{y, u}+2 \phi_{y, w}\right]}\right.\right.\right. \\
& \left.\left.\left.+e^{i\left[-\phi_{x, u}+\phi_{x, w}+2 \phi_{y, u}+2 \phi_{y, w}\right]}\right)\right]\right\} h_{y,+}^{4},
\end{aligned}
$$

where the resonance basis $h_{q, \pm}$ relates to the action-angle variables as $h_{x, \pm}=\sqrt{2 J_{x}} e^{ \pm i \Phi_{x}}$ and $h_{y, \pm}=\sqrt{2 J_{y}} e^{ \pm i \Phi_{y}}, \phi_{x, y}$ is the phase-advance at the multipole location, $b_{3}$ and $b_{4}$ are the integrated sextupole and octupole strengths, respectively.

[1] EBS storage ring technical report-ESRF, ESRF Publications, 2019.

[2] A design report of the baseline for PEP-X: An ultra-low emittance storage ring, Report No. SLAC-PUB-13999, 2010.

[3] B. Singh and H. Ghasem, Lattice options for DIAMONDII, in Proceedings for IPAC2018, THPMF009 (JACoW Publishing, Vancouver, 2018).

[4] S. Leemann, Lattice design for the MAXIV storage rings, (2016.

[5] A. Loulergue et al., Baseline lattice for the upgrade of SOLEIL, in Proceedings for IPAC2018, THPML034 (JACoW Publishing, Vancouver, 2016).

[6] K. L. Brown and R. V. Servranckx, Optics modules for circular accelerator design, Nucl. Instrum. Methods Phys. Res., Sect. A 258, 480 (1987).
[7] K. Oide and H. Koiso, Dynamic aperture of electron storage rings with noninterleaved sextupoles, Phys. Rev. E 47, 2010 (1993).

[8] R. Bartolini, Design and optimization strategies of nonlinear dynamics for diffraction limited synchrotron light sources, in Proceedings of IPAC2016, MOZA02 (Busan, Korea, 2016).

[9] A. Streun, Non-linearities in light sources, Report No. SLS-TME-TA-2005-0282, 2005.

[10] M. Borland et al., Direct methods of optimization of storage ring dynamic and momentum aperture, in Proceedings of PAC09, TH6PFP062 (JACoW Publishing, Vancouver, 2009).

[11] M. Borland et al., Multi-objective direct optimization of dynamic acceptance and lifetime for potential ugrades of the Advanced Photon Source, Report No. ANL/APS/LS319, 2009. 
[12] L. Yang, Y. Li, W. Guo, and S. Krinsky, Multiobjective optimization of dynamic aperture, Phys. Rev. ST Accel. Beams 14, 054001 (2011).

[13] Y. Li, W. Cheng, L. H. Yu, and R. Rainer, Genetic algorithm enhanced by machine learning in dynamic aperture optimization, Phys. Rev. Accel. Beams 21, 054601 (2018).

[14] O.S. Brning and J.-P. Koutchouk, Feasibility study for compensating the anharmonicities in LEP with additional octupoles, Report No. CERN-SL-97-011-AP, 1997.

[15] S. C. Leemann and A. Streun, Perspectives for future light source lattices incorporating yet uncommon magnets, Phys. Rev. ST Accel. Beams 14, 030701 (2011).

[16] J. Ögren and V. Ziemann, Compensating amplitudedependent tune-shift without driving fourth-order resonances, Nucl. Instrum. Methods Phys. Res., Sect. A 869, 1 (2017).

[17] J. Bengtsson, The sextupole scheme for the swiss light source (SLS): An analytic approach, SLS NoteNo. 9/97, PSI, 1997.

[18] K. Soutome and H. Tanaka, Higher-order formulas of amplitude-dependent tune shift caused by a sextupole magnetic field distribution, Phys. Rev. Accel. Beams 20, 064001 (2017).
[19] C. Wang, Explicit formulas for 2nd-order driving terms due to sextupoles and chromatic effects of quadrupoles, Report No. ANL/APS/LS-330, 2012.

[20] F. Schmidt, E. Forest, and E. McIntosh, Introduction to the polymorphic tracking code: Fibre bundles, polymorphic taylor types and exact tracking, Report No. CERN-SL2002-044-AP, 2002.

[21] G. Wang, T. Shaftan, V. Smaluk, N. A. Mezentsev, S. Sharma, O. Chubar, Y. Hidaka, and C. Spataro, Complex bend: Strong-focusing magnet for low-emittance synchrotrons, Phys. Rev. Accel. Beams 21, 100703 (2018).

[22] G. Wang, T. Shaftan, V. Smaluk, Y. Hidaka, O. Chubar, T. Tanabe, J. Choi, S. Sharma, C. Spataro, and N. A. Mesentsev, Complex Bend. II. a new optics solution, Phys. Rev. Accel. Beams 22, 110703 (2019).

[23] M. Borland, ELEGANT: A flexible SDDS-compliant code for accelerator simulation, https://doi.org/10.2172/761286.

[24] A. Franchi, L. Farvacque, F. Ewald, G. Le Bec, and K. B. Scheidt, First simultaneous measurement of sextupolar and octupolar resonance driving terms in a circular accelerator from turn-by-turn beam position monitor data, Phys. Rev. ST Accel. Beams 17, 074001 (2014). 